\title{
Multimodal Treatment of Resectable Gastric Cancer with Intensive Neoadjuvant Radiation Therapy: Obninsk Radiological Center Experience
}

\author{
V. Skoropad ${ }^{*}, 1$, B. Berdov ${ }^{1}$ and L. Titova ${ }^{2}$ \\ ${ }^{I}$ Department of Surgical and Combined Treatment of Abdominal Tumors, ${ }^{2}$ Department of Radiation therapy, Federal \\ State Institution "Medical Radiological Research Center" of the Russian Ministry of Health and Social Development, \\ Koroliova Street 4, 249036 Obninsk, Russia
}

\begin{abstract}
Introduction: We performed a retrospective analysis to clarify the role of neoadjuvant radiation therapy in the management of resectable gastric cancer.

Methods and Methodology: All patients underwent R0 gastrectomy between 1974 and 2008 were retrospectively evaluated. Among them 597 patients underwent multimodal treatment with various schemes of intensive preoperative radiation therapy (total dose of 20-27 Gy) and 433 patients were treated with surgery alone.

Results: Radiation therapy was completed in $98 \%$ of the patients without the delay of the surgery and any increase in postoperative morbidity or mortality including cases with D2 lymphadenectomy. Subgroup analysis showed that neoadjuvant radiotherapy provided statistically significant survival improvement comparing with surgery alone in $\mathrm{pN}+$ cases $(\mathrm{p}=0.035)$; Borrmann 3 and 4 types $(\mathrm{p}=0.029)$; poorly differentiated, undifferentiated and signet ring cell carcinomas $(\mathrm{p}=0.005)$. Multivariate analysis in the latter group confirmed that neoadjuvant radiation therapy was an independent factor of the favorable prognosis $(\mathrm{p}=0.001)$.

Conclusions: Intensive neoadjuvant radiotherapy is safe, feasible and could improve survival in selected groups of patients. Patients with poorly differentiated, undifferentiated and signet ring cell carcinomas should be considered as the target group for the multimodal treatment including radiation therapy.
\end{abstract}

Keywords: Gastric cancer, long-term results, multimodal treatment, neoadjuvant radiation therapy.

\section{INTRODUCTION}

Despite global decreasing in incidence, stomach cancer remains one of the major oncological problem. It is one of the most important causes of cancer morbidity and mortality in Russia, keeping the $2^{\text {nd }}$ place in males and the $3 \mathrm{~d}$ place in females. In 2007, 41941 new cases of gastric cancer were diagnosed and 37456 patients died [1]. There were marked changes in the treatment strategy of gastric cancer during last decades. First, gastrectomy with D2 lymphadenectomy became standard procedure not only in Japan but also in specialized Centers in Europe and the USA. Secondly, two successful randomized trials (Intergroup 0116/SWOG 9008 [2] and MAGIC [3] had lead to changing of the treatment approaches to locally advanced gastric cancer: more and more patients nowadays undergo multimodal treatment with neoadjuvant or adjuvant therapy. And finally, the results of recent randomized trial (ToGA) had opened perspectives of targeted therapy [4]. So the necessity of multimodal approaches to gastric cancer is evident and nowadays we have effective tools to improve results of surgical treatment. On the other hand there is no consensus concerning all aspects of neoadjuvant or adjuvant therapy including the best setting (pre-, intra-, post- or perioperative), the composition

*Address correspondence to this author at the Federal State Institution "Medical Radiological Research Center" of the Russian Ministry of Health and Social Development, Koroliova Street 4, 249036 Obninsk, Russia;

Tel: +7(48439) 93165; Fax: +7(495) 9561440;

E-mail: skoropad@mrrc.obninsk.ru (radio-, chemo-, targeted therapy) and the optimal sequence of treatment modalities.

Intensive neoadjuvant radiotherapy in the multimodal treatment of gastric cancer have been investigated in occasional trials and the outcome is controversial [5-13].

Medical Radiological Research Center has probably the largest experience in the world of an use of intensive preoperative radiation therapy for resectable gastric cancer. During 35 years more than 1000 patients underwent curative surgery and about 600 patients were treated with neoadjuvant radiation therapy. During these years several schemes of radiation therapy were elaborated and clinically tested. This paper is mostly focused on long-term survival and the discussion on the role of radiation therapy in future trials.

\section{MATERIALS AND METHODOLOGY}

\section{Diagnosis and Staging}

To confirm gastric cancer and to determine the resectability a number of radiographic and endoscopic modalities were used depending of the period of the study. Recently the diagnostic algorithm looks as the follows: gastroscopy with the biopsy, transabdominal ultrasound and chest $\mathrm{x}$-ray films are the obligatory procedures. Multispiral computed tomography and laparoscopy with peritoneal cytology are done for most patients except for cases with evident early gastric cancer. 


\section{Patients and Tumors Characteristics}

Between 1974 and 2008, 1030 patients underwent curative surgery for gastric cancer. Among them, 597 patients underwent multimodal treatment and 433 - surgery alone. Patients were treated with neoadjuvant irradiation when corresponding method of radiation therapy was under clinical investigation. Otherwise surgery alone as a standard treatment modality was used. Most radiotherapy trials were phase 1 or 2, and uncontrolled. The exception was our small randomized clinical trial carried out in 1974-1978 [11]. Written informed consent was obtained from all the patients.

Among the patients males predominated (618 patients); the age ranged from 19 to 89 years (median - 57 years). Tumor-related complications were registered in more than a half of the patients and included anemia (257 cases), pylorostenosis (134) and dysphagia (134). Most tumors were located in the distal part of the stomach (453 patients); involvement of gastric body (226) and cardia (268) were equal in the frequency. Most tumors presented Borrmann' 3 or 4 types (731 patients). Among tumor histology, poorly differentiated carcinoma predominated (618 cases), signet ring cell carcinoma was seen in 185 patients. The degree of the gastric wall invasion (pT stage according to the $6^{\text {th }}$ edition of UICC TNM classification) was as the follows: pT1 - 206 cases, pT2a/2b - 381 cases; pT3 - 412 cases; pT4 - 31 cases. Regional lymph node involvement was histologically confirmed in 375 patients.

\section{Radiation Therapy}

The details of neoadjuvant radiation therapy are given in Table 1. The first scheme was elaborated and applied in early $70^{\text {th }}$ of the $20^{\text {th }}$ century. The total tumor dose of $20 \mathrm{~Gy}$ was delivered over 5 consecutive days (course 1) [11]. In 198081 , we carried out a phase 1 , and in 1982-88 - a phase 2 clinical trials in order to explore the toxicity and the efficacy of the same scheme with metronidazole as a radiosensitizer (course 2) [12]. In late $80^{\text {th }}$ original uneven regime using radiobiological considerations, clinical experience and mathematical modeling and based on the conception of the total tolerate dose [14] was elaborated and studied in the prospective clinical trial (course 3) [8]. Since 2002 year, we have been using neoadjuvant radiation therapy of $25 \mathrm{~Gy}$ for 5 consecutive days with daily dose division in two equal fractions with 4-5 hours interval (course 4). Daily dose division is expected to produce less damage on normal tissues because of forthcoming major sugery. Initially patients were treated with gamma rays of Cobalt-60 using domestic "AGAT" and "ROKUS" apparatus. Since 1993, megavoltage photons from electron accelerator "Philips SL
20" were used. Two opposite fields, ranged from $10 \times 12$ to $14 \times 16 \mathrm{~cm}$, were treated. 2D planning was used, the target volume as a rule covered the whole stomach and regional lymph nodes along curvatures, celiac axis and its branches. Because of the long period of the study and an use of different, mostly domestic radiotherapeutic machines radiotherapy planning in most patients were not made according to ICRU.

\section{Surgery}

Subtotal distal gastrectomy was performed for distally located lesions when proximal tumor-free margin of 3 to 5 $\mathrm{cm}$ depending of the tumor macroscopic type and morphology could be achieved (566 patients). Subtotal proximal gastrectomy was a rare operation reserved for small tumors located around gastric cardia (52 patients). Total gastrectomy was undertaken for other tumors (412 patients). In all patients complete dissection of perigastric and left gastric artery lymph nodes was performed (D1+N 7). Since 2000 year, systemic D2 dissection became the standard operation for all cases except for palliative surgery and evident early gastric cancer. Resection of other organs was performed when the direct tumor invasion or metastatic disease were suspected (19\% of the patients).

\section{Statistical Analysis}

The statistical analysis was conducted using Prism 3.0 software system (GraphPad Software, Inc., San Diego, USA). The chi- square test or Fisher's exact test were used to determine whether the distribution of prognostic variables was significantly different. Continuous data were evaluated with the Student $t$ test. The survival was calculated from the time of surgery by the Kaplan-Meier method. The end points were death as a result of any cause (overall survival). Patients, lost to follow up, were censored at the time of last observation. Comparisons of survival curves were made with the log-rank test. For multivariate analysis Cox's proportional hazards model or logistic regression model were used. For all tests, a two-tailed $\mathrm{P}$ value of $<0.05$ was considered to be statistically significant.

\section{RESULTS}

\section{Radiation-Related Toxicity}

Preoperative radiotherapy was generally well tolerated; it was completed without any delay of surgery in 587 out of 597 cases (98\%). Regimes with daily dose division were best tolerated (completed in 99\%). Any side effects developed in $322(54 \%)$ patients, gastrointestinal toxicity predominated. Grade 1-2 (according to NCIC CTG specific criteria,

Table 1. Schemes of Neoadjuvant Radiation Therapy

\begin{tabular}{|c|c|c|c|c|c|c|c|c|c|c|c|c|c|}
\hline \multirow{2}{*}{ Course } & \multirow{2}{*}{ No of Patients } & \multicolumn{11}{|c|}{ Day of the Week, Dose Per Fraction (Gy) } & \multirow{2}{*}{$\begin{array}{l}\text { Interval to the } \\
\text { Operation, Days }\end{array}$} \\
\hline & & Fr. & Su. & So. & Mo. & Tu. & Wen. & Thur. & Fr. & Su. & So. & Mo. & \\
\hline 1 & 312 & - & - & - & 4 & 4 & 4 & 4 & 4 & - & - & - & $3-5$ \\
\hline 2 & 56 & - & - & - & $4+\mathrm{MZ}$ & 4 & $4+\mathrm{MZ}^{*}$ & 4 & $4+\mathrm{MZ}$ & - & - & - & $3-5$ \\
\hline 3 & 176 & $3+4$ & - & - & $1.5+3.5$ & $1+1.5$ & $1+1.5$ & $1+1.5$ & $1+1.5$ & - & - & $1.5+3.5$ & $1-3$ \\
\hline 4 & 53 & - & - & - & $2.5+2.5$ & $2.5+2.5$ & $2.5+2.5$ & $2.5+2.5$ & $2.5+2.5$ & - & - & - & $3-5$ \\
\hline
\end{tabular}


EORTC version, 1994) nausea, weakness, loss of appetite $(28 \%)$ and vomiting $(24 \%)$ were seen most frequently. No haematological toxicity was registered. During recent 10 years adverse events developed significantly rarely - in 31 (18\%) cases which may be explained by an use of electron accelerators instead of Cobalt-60 machines.

\section{Postoperative Morbidity and Mortality}

Postoperative morbidity and mortality, which was defined as death during a hospital stay or 30-days period, were calculated for the whole 35 -years period as well as for eight recent years when all D2 operations were performed. The latter data seem to be most important as they reflect the current outcome of gastric cancer surgery and may be compared to modern literature.

During 35-years period postoperative complications were registered in $330(32 \%)$ patients with the similar frequency in multimodally treated and surgically treated groups. During 8 recent years the complications rate has decreased significantly from $43 \%$ to $12.5 \%(\mathrm{p}=0.002)$. The similar situation was seen with postoperative mortality: it has decreased from $4.8 \%$ to $1.9 \%(\mathrm{p}=0.03)$. Table 2 shows details of most important complications and mortality after total and distal subtotal gastrectomy performed during 8 recent years. Anastomotic leakage is the most dramatic complication after total gastrectomy; it developed in 2.8$4.8 \%$ of patients. It would be underlined that in most cases it was asymptomatic and was revealed by the radiological examination routinely performed on the $6^{\text {th }}$ day after surgery. In these cases the parentheral nutrition was prolonged for an additional week and no other action was done. Other complications were seen in occasional cases independently of the treatment modality. The only significant difference between treatment groups was lower incidence of postoperative pancreatitis in radiation therapy group. This phenomena was described in our previous studies $[10,11]$. No patients experienced pancreatic necrosis. There were 3 postoperative deaths due to abdominal abscess, anastomotic leakage and stroke. Subtotal distal gastrectomy was much safer operation with overall complication rate of $10.7 \%$ and mortality rate of $1.25 \%$. Deaths were caused by anastomotic leakage (1) and myocardial infarction (1). To identify independent prognostic factors associated with postoperative mortality we performed multivariate analysis using logistical regression model. The analysis revealed three factors: the period when the operation was performed, patient's age and the type of the procedure. The mortality was significantly lower during last decade, in patients younger than 55 years and when subtotal distal gastrectomy was used. Neither neoadjuvant radiation therapy nor D2 dissection influenced it.

\section{Survival}

As our study was a retrospective one, we have focused on the subgroup survival analysis. All 1030 patients were included in the analysis.

Table 3 shows univariate analysis of 5-year overall survival after multimodal and surgical treatment. The treatment results of tumors which did not penetrate the serosal layer and had distal location were acceptable with 5year survival of about $60 \%$. The results worsened significantly in pT3 cases and proximal location and became extremely low when there was entire stomach or gastric stump involvement. When regional lymph nodes were negative neoadjuvant radiation therapy did not provide any survival benefit. In contrast multimodal treatment had advantage over surgical treatment in $\mathrm{N}$-positive cases $(\mathrm{p}=0.035)$. Patients' gender and age did not influence the survival significantly although multimodal treatment provided borderline benefit to males and patients older than 55 years. Patients with macroscopic Borrmann 1-2 types did relatively well after both treatment approaches while multimodal treatment was significantly superior in Borrmann 3-4 types tumors $(\mathrm{p}=0.03)$. Subtotal distal gastrectomy

Table 2. Postoperative Morbidity and Mortality in 2000-2008 Years

\begin{tabular}{|c|c|c|c|c|}
\hline \multirow{2}{*}{ Complication } & \multicolumn{2}{|c|}{ Total Gastrectomy, No = 112} & \multicolumn{2}{|c|}{ Subtotal Distal Gastrectomy, No = 159} \\
\hline & $\begin{array}{c}\text { RT+Surgery } \\
\text { No }=71\end{array}$ & $\begin{array}{c}\text { Surgery } \\
\text { No=41 }\end{array}$ & $\begin{array}{c}\text { RT+Surgery } \\
\text { No }=67\end{array}$ & $\begin{array}{c}\text { Surgery } \\
\text { No }=92\end{array}$ \\
\hline Anastomotic leakage & $2(2.8)^{*}$ & $2(4.8)$ & 0 & $1(1.1)$ \\
\hline Duodenal stump leakage & 0 & 0 & 0 & 0 \\
\hline Abdominal abscess & $1(1.4)$ & 0 & 0 & 0 \\
\hline Small bowel obstruction & 0 & 0 & $1(1.5)$ & $1(1.1)$ \\
\hline Pancreatitis & 0 & $3(7.3)$ & 0 & $2(2.2)$ \\
\hline Pancreatic necrosis & 0 & 0 & 0 & 0 \\
\hline Anastomotic / other bleeding & 0 & 0 & $1(1.5)$ & 0 \\
\hline Pulmonary embolism & 0 & $1(2.4)$ & 0 & 0 \\
\hline Myocardial infarction & 0 & 0 & 0 & $1(1.1)$ \\
\hline Pneumonia & $4(5.6)$ & $2(4.8)$ & $3(4.4)$ & $2(2.2)$ \\
\hline Total $\%$ of complications & $9(12.6)$ & $8(19.5)$ & $7(10.4)$ & $10(10.9)$ \\
\hline Mortality & $2(2.8)$ & $1(2.4)$ & 0 & $2(2.1)$ \\
\hline
\end{tabular}

*-In parentheses are given percentages. 
Table 3. Univariate Analysis of 5-Year Survival (\%) After Multimodal and Surgical Treatment

\begin{tabular}{|c|c|c|c|}
\hline Variables & Radiation Therapy + Surgery & Surgery Alone & $P$ Value \\
\hline $\mathrm{pT} 1 / \mathrm{T} 2 \mathrm{a} / \mathrm{T} 2 \mathrm{~b}$ & $60 \pm 3$ & $61 \pm 3$ & 0.92 \\
\hline pT3 & $42 \pm 4$ & $30 \pm 4$ & 0.06 \\
\hline $\mathrm{pN} 0$ & $61 \pm 3$ & $60 \pm 3$ & 0.51 \\
\hline $\mathrm{pN}+$ & $37 \pm 4$ & $25 \pm 4$ & 0.035 \\
\hline Proximal location & $46 \pm 4$ & $36 \pm 4$ & 0.34 \\
\hline Central and distal location & $61 \pm 3$ & $55 \pm 3$ & 0.25 \\
\hline Males & $53 \pm 3$ & $46 \pm 4$ & 0.06 \\
\hline Females & $52 \pm 4$ & $50 \pm 4$ & 0.8 \\
\hline Age $<55$ & $53 \pm 3$ & $52 \pm 3$ & 0.95 \\
\hline Age $\geq 55$ & $52 \pm 3$ & $43 \pm 4$ & 0.058 \\
\hline Borrmann 1-2 types & $66 \pm 4$ & $67 \pm 4$ & 0.7 \\
\hline Borrmann 3-4 types & $41 \pm 3$ & $33 \pm 3$ & 0.03 \\
\hline Total gastrectomy & $40 \pm 4$ & $30 \pm 5$ & 0.046 \\
\hline Subtotal distal gastrectomy & $61 \pm 3$ & $57 \pm 3$ & 0.48 \\
\hline Well and moderately differentiated carcinomas & $51 \pm 4$ & $53 \pm 4$ & 0.46 \\
\hline Poorly-, undifferentiated and signet ring cell carcinomas & $54 \pm 3$ & $41 \pm 4$ & 0.005 \\
\hline
\end{tabular}

resulted in a good long-term survival in both groups. Total gastrectomy was done for larger and more proximally located as well as diffuse tumors, thus much poorer longterm results were obtained and there was a benefit in favor of neoadjuvant radiation therapy. The long-term results of well and moderately differentiated tumors were equal independently of the treatment modality. In contrast multimodal treatment of poorly differentiated, undifferentiated and signet ring cell carcinomas had significant advantages over surgical treatment (0.005).

Taking into consideration the above mentioned results we have focused the further analysis on patients having poorly and undifferentiated tumors (Table 4). Univariate analysis has shown that significant survival advantage of multimodal treatment was seen except for $\mathrm{pT} 1$ cases and 1 stage $(\mathrm{p}=0.9)$. The highest level of significance was reached for undifferentiated and signet ring cell carcinomas. Multivariate analysis using Cox proportional hazard model was performed among patients with poorly, signet ring cell and undifferentiated tumors (618 patients). Four factors were identified to have independent prognostic significance among this group: tumor location (distal vs proximal, $\mathrm{p}=0.019), \mathrm{pT}(\mathrm{pT} 1-2$ vs $\mathrm{pT} 3-4, \mathrm{p}=0.018), \mathrm{pN}$ (pN0 vs pN1-3, $\mathrm{p}=0.001$ ) and treatment modality (multimodal treatment $v s$ surgical treatment, $\mathrm{p}=0.001$ ). Thus the treatment modality is an independent prognostic factor; its influence is high and comparable with that of lymph node status.

\section{DISCUSSION}

Despite decreasing in incidence gastric cancer remains one of the leading causes of cancer death worldwide. Oneyear mortality in Russia is as high as 53\% [1] which reflects not only late diagnosis but poor treatment results as well. High recurrence rate even after potentially curative operations shows that surgery as a single treatment of locally advanced gastric cancer is insufficient. Most recent publications reflect the movement toward multimodality therapy of gastric cancer including radiotherapy [15-17] but surgery still remains most frequently used single-treatment modality. Among patients treated with curative intent in Russia in 2007, 85\% cases underwent surgery alone although stage 1 of the disease was diagnosed in less than $15 \%$ of them [1].

Stomach carcinoma has been traditionally considered as a radioresistant tumor. On the other hand some authors reported high radiosesitivity of poorly differentiated and signet-ring cell tumors [18]. Intensive preoperative irradiation has not a goal to destroy the tumor completely or to achieve marked regression. The aim is to decrease the biological potential of tumor cells which may be disseminated during surgical procedure and hence to increase the operation radicality as well as to eradicate subclinical micrometastases. Recent publications show that the operative procedure including lymph node dissection itself is an important factor of cancer cells dissemination and further tumor relapse $[19,20]$. Despite relatively long history, the outcome of intensive preoperative radiotherapy for gastric and gastroesophageal junction tumors remains controversial.

Nakayama et al. [5] were one of the first who used concentrated radiation therapy for esophageal and proximal gastric cancer. The total dose of $20 \mathrm{~Gy}$ was delivered over 3 days ( 7 - 7 - 6 Gy per day), a single fraction of 10 Gy was used as well. The authors analyzed radiation-induced tumor damage and concluded that the interval of 4-7 days after radiation therapy is the optimal to perform the surgery. A number of trials including several randomized ones had been curried out in the former USSR [6-12]. Our early small randomized trial showed good tolerance of intensive 
Table 4. Univariate Analysis of 5-Year Survival (\%) of Patients with Poorly Differentiated, Signet Ring Cell and Undifferentiated Carcinoma After Multimodal and Surgical Treatment

\begin{tabular}{|c|c|c|c|}
\hline Variables & Radiation Therapy + Surgery & Surgery Alone & \multicolumn{2}{|c|}{$41 \pm 4$} \\
\hline \hline PDC, SRCC, UDC & $54 \pm 3$ & $36 \pm 4$ & 0.005 \\
\hline PDC, SRCC, UDC; pT2-4 & $50 \pm 3$ & $53 \pm 5$ & 0.007 \\
\hline PDC, SRCC, UDC; pN0 & $63 \pm 4$ & $23 \pm 5$ & $32 \pm 7$ \\
\hline PD, SRCC, UDC; pN+ & $37 \pm 4$ & $27 \pm 8$ & 0.01 \\
\hline SRCC & $53 \pm 5$ & $31 \pm 5$ & 0.006 \\
\hline UDC & $55 \pm 9$ & 0.017 \\
\hline SRCC, UDC & $54 \pm 4$ & 0.000 \\
\hline
\end{tabular}

PDC - poorly differentiated carcinoma.

SRCC - signet ring cell carcinoma.

UDC - undifferentiated carcinoma.

radiotherapy with no increase of postoperative morbidity and mortality rates compared to surgery alone although no significant difference in overall survival was detected between the two treatment groups after 20-year of follow-up [11]. Schepotin et al. [9] reported that preoperative intensive radiation therapy $(20$ Gy / 5 fractions $)$ with local hyperthermia followed by surgery resulted in a significant improvement in 3- and 5-year survival of 21-22\% comparing with surgery alone or surgery plus radiation therapy without hyperthermia. Two multicenter randomized trials were conducted to compare concentrated preoperative radiotherapy (20Gy/5 fractions) with surgery alone for tumors located in the proximal part [6] and the distal part [7] of the stomach. Vashakmadze et al. [6] failed to show survival advantages of preoperative radiation therapy, 5-year survival was similar in both groups $(10 \%$ and $12 \%)$. On the other hand the authors noted that most disease recurrences were registered during the $1^{\text {st }}$ and the $2 \mathrm{~d}$ years after surgical treatment and during the $3 \mathrm{~d}$ and the $4^{\text {th }}$ years after multimodal treatment and concluded that preoperative radiotherapy prolonged recurrence-free survival. Talaev et al. [7] reported that multimodal treatment resulted in significantly better survival at 3 years $(64 \%$ vs $48 \%)$ and at 5 years $(52 \%$ vs $35 \%)$ for the tumors located in gastric body and antrum $(p<0.05)$. It should be noted that in above mentioned studies no statistical tests comparing entire survival curves were used. The difference between treatment groups was calculated using Student's t test at fixed terms - 3 and 5 years.

Obninsk Radiological Research Center has the largest experience in the world in multimodal treatment of gastric cancer with intensive preoperative radiotherapy. Our own results as well as available literature data demonstrate that intensive courses of preoperative radiotherapy are well tolerated, may be completed in most patients and do not influence postoperative morbidity and mortality even in combination with D2 surgery [13]. Thus this treatment modality is safe and feasible. It is much more difficult to demonstrate survival advantages of any new treatment modality over the standard one. We can remind that two large European randomized trials failed to show any benefit of D2 resection but nowadays it is recognized as the "golden standard" of gastric cancer surgery.
In this paper the authors present a retrospective study and do completely accept the skepticism concerning such kind of analysis. As it was expected tumor stage as well as pT and pN parameters were important prognostic factors independently of the treatment modality used. However it should be noted that all of these parameters are more

pathological than clinical ones and we can exactly identify them only after the operation and the histological examination of the resected specimen. On the other hand the indication for preoperative therapy must be accessible before the operation and tumor morphology meets this requirement.

It is controversial whether tumor histology has influence treatment strategy and patient's outcome although some authors noted that patients with poorly differentiated tumors had worse prognosis [7, 21, 22]. In our study the most impressive survival advantages of preoperative radiotherapy were obtained in patients with poorly differentiated, undifferentiated and signet ring cell carcinomas (Tables $\mathbf{3}$ and 4). As to our mind these findings are very important because during recent decades there exists the increase in these types of gastric cancer especially - in signet ring cell carcinomas. In our Center the proportion among curatively operated patients with signet-ring cell carcinomas or undifferentiated carcinomas in 1974-1980 was 6 and 9\% (in total $-15 \%$ ), and in 1998-2008 - 10 and $22 \%$ (in total $32 \%)$, respectively $(\mathrm{p}=0.03)$. Nered et al. [22] conducted a large retrospective analysis and reported that $\mathrm{D} 2$ gastrectomy comparing with D1 procedure was associated with significant survival advantages in patients with differentiated tumors. On the other hand it appeared that D2 dissection did not improve survival in patients with poorly differentiated and signet-ring cell carcinomas. Another word that was the cases when preoperative radiotherapy has demonstrated survival advantages over surgery alone in our study.

Unfortunately there is extremely small number of well organized randomized trials tested preoperative radiotherapy. Fiorica F, et al. [23] have recently published a meta-analysis on the impact of radiation therapy on survival in resectable gastric cancer. They have found only four eligible randomized trials testing preoperative irradiation (832 patients) including two our small trials. However based on the published data the authors concluded that preoperative 
radiotherapy (as well as postoperative chemo-radiotherapy) significantly reduced 3-year and 5-year overall mortality comparing with surgery alone. It is clear that further randomized trials are needed to clarify the impact of preoperative radiotherapy and chemo-radiotherapy on survival and to determine the group of potential "responders" who may have the survival benefit.

\section{CONCLUSION}

In conclusion, intensive neoadjuvant radiotherapy allows the delivery of a moderate dose of radiation during short time without surgery delay. It is safe, feasible and does not increase postoperative morbidity and mortality even in the case of extended surgery. Multimodal treatment could improve survival in selected groups of patients mostly with poorly differentiated, undifferentiated and signet ring cell carcinomas. The above mentioned group of patients represents the target group to be included in future neoadjuvant radiation (chemo-radiation) therapy trials.

\section{REFERENCES}

[1] Chissov VI, Starinsky VV. Malignant tumors in Russia in 2007 (incidence and mortality). Moscow, 2009.

[2] Macdonald JS, Smalley SR, Benedetti J, et al. Chemoradiotherapy after surgery compared with surgery alone for adenocarcinoma of the stomach or gastroesophageal junction. N Engl J Med 2001; 345: 725-30.

[3] Cunningham D, Allum WH, Stenning SP, et al. Perioperative chemotherapy versus surgery alone for resectable gastroesophageal cancer. N Engl J Med 2006; 355; 11-20.

[4] Van Cutsem E, Kang Y, Chung H, et al. Efficacy results from the ToGA trial: A phase III study of trastuzumab added to standard chemotherapy (CT) in first-line human epidermal growth factor receptor 2 (HER2)-positive advanced gastric cancer (GC). J Clin Oncol 2009; 27:18s, (suppl; abstr LBA4509).

[5] Nakayama K, Yanagisawa F, Nabeya K, et al. Concentrated preoperative irradiation therapy. Arch Surg 1963; 87: 1003-18.

[6] Vashakmadze LA, Babaian LA, Talaev MI, Koliadiuk IV, Savinov VA. Results of the combined treatment of cancer of the cardial region of the stomach (a cooperative, randomized study). Vopr Onkol 1987; 33: 70-4.

[7] Talaev MI, Starinsky VV, Kovalev BN, et al. Results of combined treatment for cancer of the antral part and the body of the stomach. Voprosy Onkol 1990; 36: 1485-8.

[8] Skoropad VY, Berdov BA, Mardynsky YS. Preoperative irradiation in the dynamic dose fractionation regime in combined therapy of stomach cancer. Med Radiol 1992; 7-8: 33-6.
[9] Shchepotin IB, Evans SR, Chorny V, et al. Intensive preoperative radiotherapy with local hyperthermia for the treatment of gastric carcinoma. Surg Oncol 1994; 3: 37-44.

[10] Skoropad V, Berdov B, Mardynsky Yu, Titova L. A prospective, randomized trial of pre-operative and intraoperative radiotherapy $v s$ surgery alone in resectable gastric cancer. Eur J Surg Oncol 2000; 26: 773-9.

[11] Skoropad V, Berdov B, Zagrebin V. Concentrated preoperative radiotherapy for resectable gastric cancer: 20-years follow-up of a randomized trial. J Surg Oncol 2002; 80: 72-8.

[12] Skoropad V, Berdov B., Zagrebin V. Preoperative radiation therapy in combination with metronidazole for resectable gastric cancer: long-term results of a phase 2 study. Eur J Surg Oncol 2003; 29: $167-71$.

[13] Skoropad V, Berdov B, Mardynsky Yu, Titova L, Pakhomenko K. Preoperative and intraoperative radiotherapy in combination with extended lymphadenectomy in the treatment of locally-advanced gastric cancer: results of phase 2 clinical trial. Vopr Onkol 2004; 50: 585-9.

[14] Ivanov VK, Petrovsky AM. Maximum potentialities of tumor radiotherapy (concept of the total tolerate dose). Med Radiol 1981; 3: 36-9.

[15] Apisarnthanarax S, Tepper JE. Crossroads in the combinedmodality management of gastroesophageal junction carcinomas. Gastrointest Cancer Res 2008; 2: 235-43.

[16] Krishnan S. The role of adjuvant radiation therapy in nonmetastatic gastric cancer: an evolving paradigm. Gastrointest Cancer Res 2009; 3: 33-5.

[17] McCloskey SA, Yang GY. Benefits and challenges of radiation therapy in gastric cancer: techniques for improving outcomes. Gastrointest Cancer Res 2009; 3: 15-9

[18] Belous TA, Frank GA, Repina AG, Daryalova SL. Pathomrpfosis of stomach cancer following preoperative radiation therapy after the dynamic fractionation scheme. Med Radiol 1992; 2: 41-4.

[19] Miyazono F, Natsugoe S, Takao S, et al. Surgical maneuvers enhance molecular detection of circulating tumor cells during gastric cancer surgery. Ann Surg 2001; 233: 189-94.

[20] Marutsuka T, Shimada S, Shiomori K, et al. Mechanisms of peritoneal metastasis after operation for non-serosa-invasive gastric carcinoma: an ultrarapid detection system for intraperitoneal free cancer cells and a prophylactic strategy for peritoneal metastasis. Clin Cancer Res 2003; 9: 678-85.

[21] Rohatgi PR, Yao JC, Hess K, et al. Outcome of gastric cancer patients after successful gastrectomy: influence of the type of recurrence and histology on survival. Cancer 2006; 107: 2576-80.

[22] Nered SN, Klimenkov AA. Surgery for gastric carcinoma in patients with high risk of peritoneal metastases. Vopr Onkol 2005; 51: 75-80.

[23] Fiorica F, Cartei F, Enea M, et al. The impact of radiotherapy on survival in resectable gastric carcinoma: a meta-analysis of literature data. Cancer Treat Rev 2007; 33: 729-40. 